\title{
UNIVERSITYOF BIRMINGHAM University of Birmingham
}

\section{Erratum: "Standing on the Shoulders of Dwarfs: The Kepler Asteroseismic LEGACY Sample. I. Oscillation Mode Parameters" (2017, ApJ, 835, 172)}

Lund, Mikkel N.; Silva Aguirre, Víctor; Davies, Guy R.; Chaplin, William J.; Christensendalsgaard, Jørgen; Houdek, Günter; White, Timothy R.; Bedding, Timothy R.; Ball, Warrick H.; Huber, Daniel; Antia, H. M.; Lebreton, Yveline; Latham, David W.; Handberg, Rasmus; Verma, Kuldeep; Basu, Sarbani; Casagrande, Luca; Justesen, Anders B.; Kjeldsen, Hans; Mosumgaard, Jakob R.

DOI:

10.3847/1538-4357/aa9658

License:

None: All rights reserved

\section{Document Version}

Publisher's PDF, also known as Version of record

Citation for published version (Harvard):

Lund, MN, Silva Aguirre, V, Davies, GR, Chaplin, WJ, Christensen-dalsgaard, J, Houdek, G, White, TR, Bedding, TR, Ball, WH, Huber, D, Antia, HM, Lebreton, Y, Latham, DW, Handberg, R, Verma, K, Basu, S, Casagrande, L, Justesen, AB, Kjeldsen, H \& Mosumgaard, JR 2017, 'Erratum: "Standing on the Shoulders of Dwarfs: The Kepler Asteroseismic LEGACY Sample. I. Oscillation Mode Parameters" (2017, ApJ, 835, 172)', The Astrophysical Journal, vol. 850, no. 1, 110. https://doi.org/10.3847/1538-4357/aa9658

Link to publication on Research at Birmingham portal

Publisher Rights Statement:

Checked for eligibility: 18/07/2019

This document appears in its final form in Astrophysical Journal, copyright @ 2017. The American Astronomical Society. All rights reserved. The final Version of Record can be found at: https://iopscience.iop.org/article/10.3847/1538-4357/aa9658

https://doi.org/10.3847/1538-4357/aa9658

\section{General rights}

Unless a licence is specified above, all rights (including copyright and moral rights) in this document are retained by the authors and/or the copyright holders. The express permission of the copyright holder must be obtained for any use of this material other than for purposes permitted by law.

- Users may freely distribute the URL that is used to identify this publication.

- Users may download and/or print one copy of the publication from the University of Birmingham research portal for the purpose of private study or non-commercial research.

- User may use extracts from the document in line with the concept of 'fair dealing' under the Copyright, Designs and Patents Act 1988 (?) - Users may not further distribute the material nor use it for the purposes of commercial gain.

Where a licence is displayed above, please note the terms and conditions of the licence govern your use of this document.

When citing, please reference the published version.

Take down policy

While the University of Birmingham exercises care and attention in making items available there are rare occasions when an item has been uploaded in error or has been deemed to be commercially or otherwise sensitive.

If you believe that this is the case for this document, please contact UBIRA@lists.bham.ac.uk providing details and we will remove access to the work immediately and investigate.

Download date: 26. Apr. 2023 


\section{Erratum: "Standing on the Shoulders of Dwarfs: The Kepler Asteroseismic LEGACY Sample. I. Oscillation Mode Parameters" (2017, ApJ, 835, 172)}

Mikkel N. Lund $^{1,2}$ (D), Víctor Silva Aguirre $^{2}$ (D) Guy R. Davies ${ }^{1,2}$ (D), William J. Chaplin ${ }^{1,2}$ (D), Jørgen Christensen-Dalsgaard ${ }^{2}$ (D), Günter Houdek $^{2}$ (D), Timothy R. White ${ }^{2}$ (D), Timothy R. Bedding ${ }^{2,3}$ (D), Warrick H. Ball ${ }^{4,5}$ (D), Daniel Huber ${ }^{2,3,6}$ (D), H. M. Antia ${ }^{7}$ (D), Yveline Lebreton $^{8,9}$, David W. Latham ${ }^{10}$ (D), Rasmus Handberg ${ }^{2}$, Kuldeep Verma ${ }^{2,7}$ (D), Sarbani Basu ${ }^{11}$ (D), Luca Casagrande ${ }^{12}$ (iD),

Anders B. Justesen ${ }^{2}$, Hans Kjeldsen ${ }^{2}$, and Jakob R. Mosumgaard ${ }^{2}$

${ }^{1}$ School of Physics and Astronomy, University of Birmingham, Edgbaston, Birmingham, B15 2TT, UK; mikkeln1@phys.au.dk

${ }^{2}$ Stellar Astrophysics Centre, Department of Physics and Astronomy, Aarhus University, Ny Munkegade 120, DK-8000 Aarhus C, Denmark

${ }^{3}$ Sydney Institute for Astronomy (SIfA), School of Physics, University of Sydney, NSW 2006, Australia

${ }^{4}$ Institut für Astrophysik, Georg-August-Universität Göttingen, Friedrich-Hund-Platz 1, 37077, Göttingen, Germany

${ }^{5}$ Max-Planck-Institut für Sonnensystemforschung, Justus-von-Liebig-Weg 3, 37077, Göttingen, Germany

${ }^{6}$ SETI Institute, 189 Bernardo Avenue, Mountain View, CA 94043, USA

${ }^{7}$ Tata Institute of Fundamental Research, Homi Bhabha Road, Mumbai 400005, India

${ }^{8}$ Observatoire de Paris, GEPI, CNRS UMR 8111, F-92195 Meudon, France

${ }^{9}$ Institut de Physique de Rennes, Université de Rennes 1, CNRS UMR 6251, F-35042 Rennes, France

${ }^{10}$ Harvard-Smithsonian Center for Astrophysics, 60 Garden Street Cambridge, MA 02138 USA

${ }^{11}$ Department of Astronomy, Yale University, PO Box 208101, New Haven, CT 06520-8101, USA

${ }^{12}$ Research School of Astronomy and Astrophysics, Mount Stromlo Observatory, The Australian National University, ACT 2611, Australia Received 2017 August 21; revised 2017 September 28; published 2017 November 21

Supporting material: machine-readable tables

In this erratum, we provide corrected sets of $r_{01,10,02}$ difference ratio values and associated uncertainties, which were overestimated in the original paper (as noted by Roxburgh 2017) due to a missing trimming in the post-processing of the Markov chain Monte Carlo (MCMC) chains for these values. The typical reduction in the ratio uncertainties from performing the trimming is a factor of 10 (see Figure 3). Other parameters optimized in the peak-bagging (for instance, individual mode frequencies) are unaffected, as the trimming was performed for these in the original work (Lund et al. 2017). We also provide updated values for the $\Delta_{2} \nu$ values of $l=3$ modes. We note that the values presented here, as with those presented in the original work, are obtained from a single peakbagging procedure (see Lund et al. 2017 for details) and have yet to be verified by independent analyses using the same input power spectra. Examples of the updated tables from the original paper are given in Tables 1-3. We note that tables with individual mode parameters (Table 2) have been added for completeness, but the parameters in these tables are unchanged compared to the original paper.

In addition to the corrected values mentioned above, we provide covariance matrices for the mode frequencies, frequency difference ratios $\left(r_{01,10,02}\right)$, and second differences $\left(\Delta_{2} \nu\right)$ for the LEGACY sample (Lund et al. 2017), which were not published with the original work. The values provided by this erratum will be available in the online version of the paper.

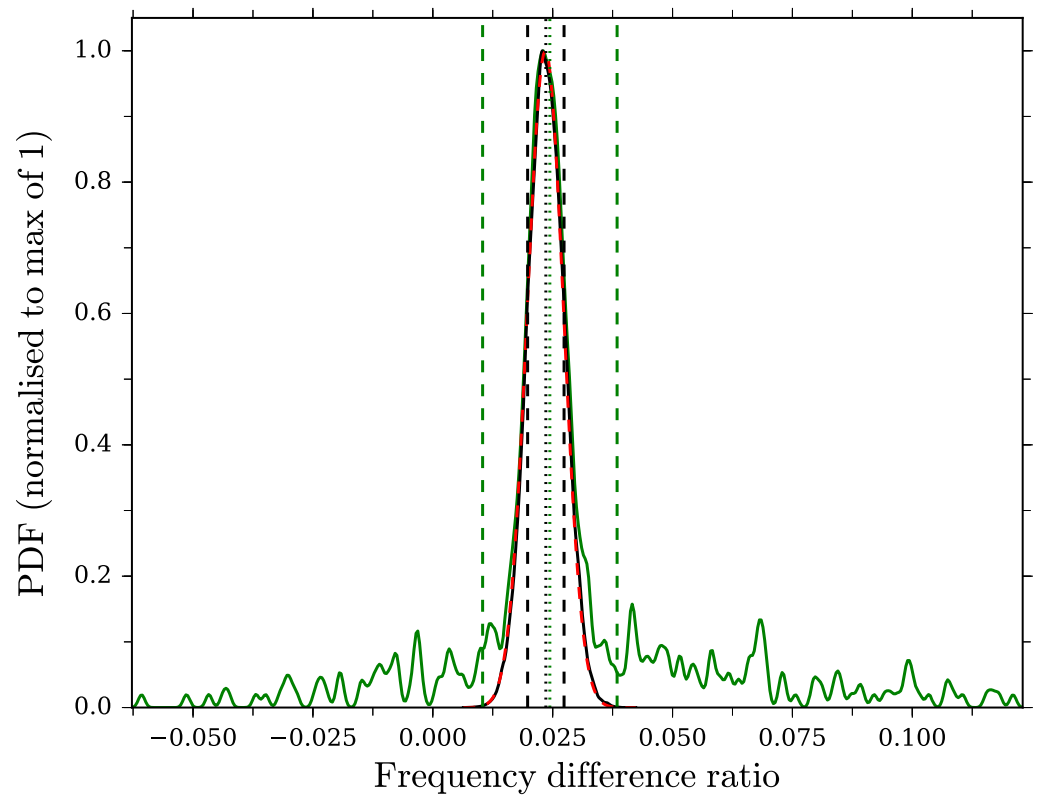

Figure 1. Comparison between ratio distribution of $r_{01, n=25}(\nu \approx 3090 \mu \mathrm{Hz}$ ) for KIC 9139151 from the full (green) and properly thinned MCMC chains (black). The dashed red line (on top of the black curve) shows the distribution obtained by sampling from the reported frequency values and corresponding uncertainties (assuming that these are normally distributed and uncorrelated). The central peak is captured by both distributions, but the wide background signal representing the ratio prior has disappeared from the thinned chains. Dotted lines indicate the distribution medians; dashed lines bound the corresponding 68\% highest probability density intervals. 

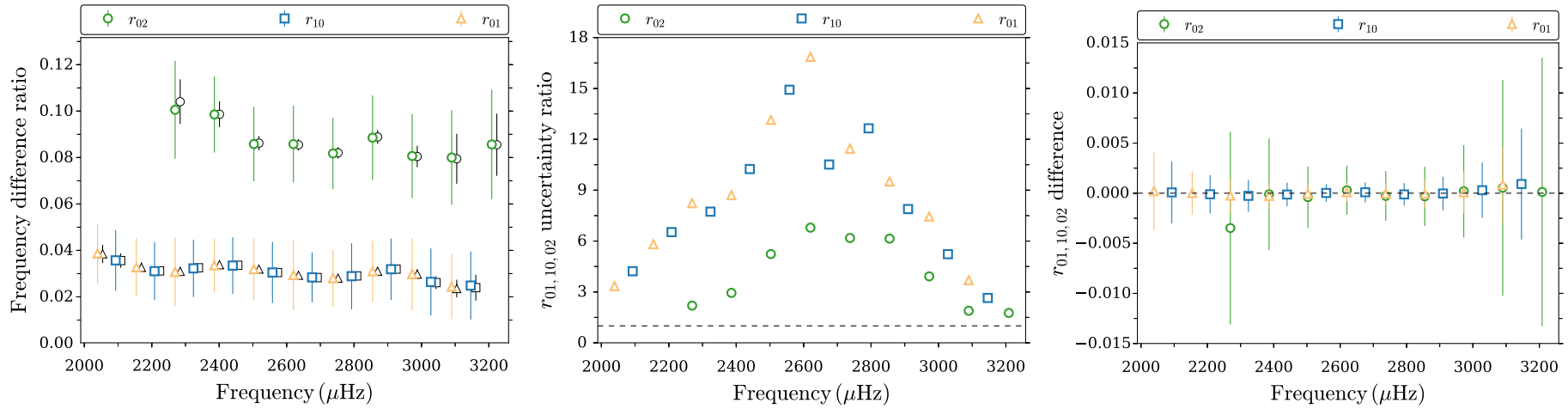

Figure 2. Left: a comparison between ratios $r_{01,10,02}$ for KIC 9139151 using the full (colored) and properly thinned MCMC chains (black). The ones from the thinned chains have been shifted $15 \mu \mathrm{Hz}$ up in frequency for clarity. Middle: the ratio between the uncertainties on $r_{01,10,02}$ for KIC 9139151 using the original and properly thinned MCMC chains. Right: the difference between central ratio values (given by the distribution median), as $r_{\text {ori }}-r_{\text {thin }}$, for KIC 9139151 . The uncertainties given here are the reduced ones obtained using the properly thinned chains.
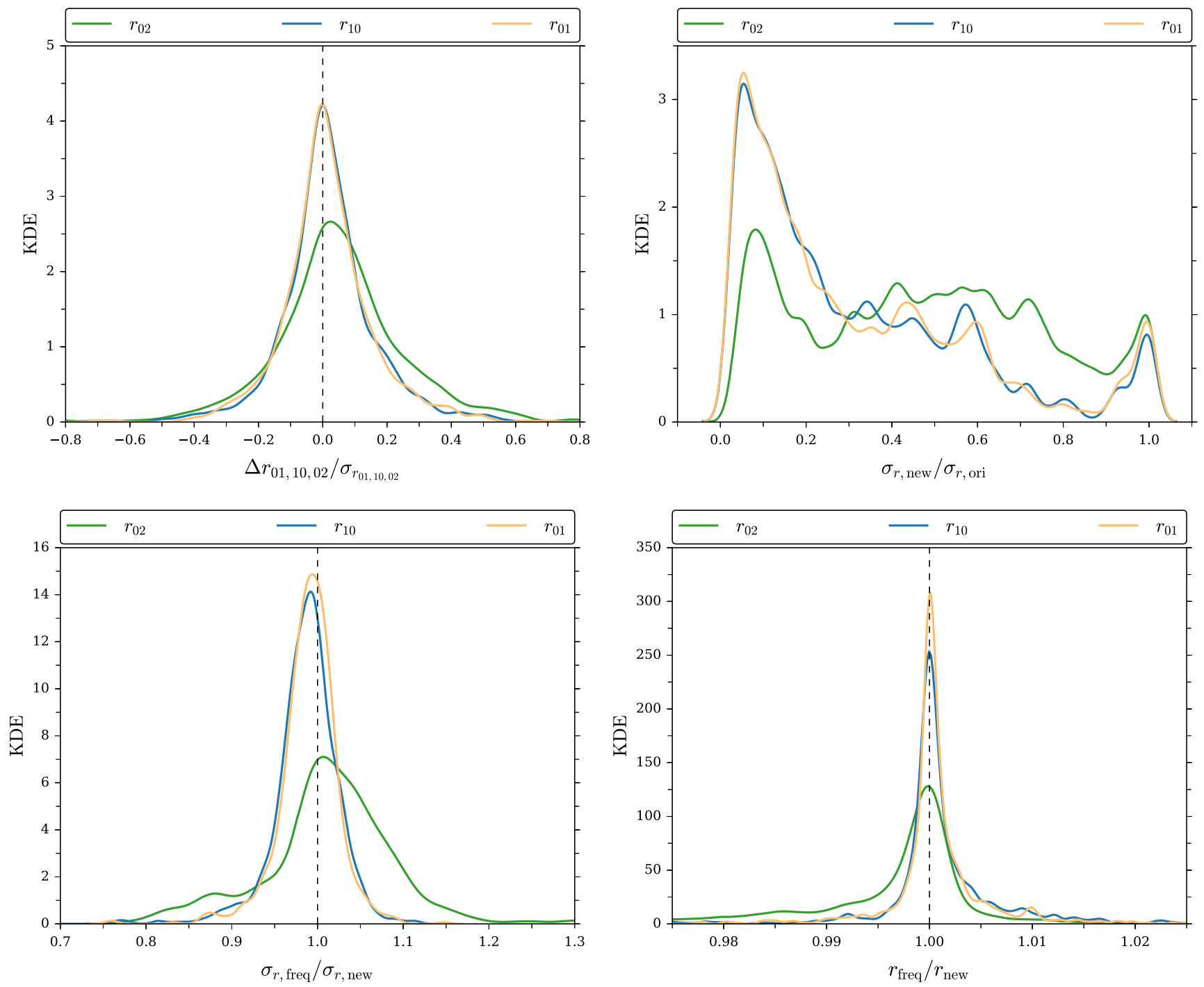

Figure 3. Top left: the distribution for the change in central ratio values $\left(\Delta r_{01,10,02}=r_{\text {ori }}-r_{\text {new }}\right)$ over the newly estimated ratio uncertainties for all LEGACY targets. Top right: the distribution for the ratio of newly estimated ratio uncertainties from the properly thinned chains over the original estimates. Bottom left: the distribution for the ratio between ratio uncertainties calculated using properly thinned chains $\left(\sigma_{\mathrm{r}, \text { new }}\right)$ and those propagated from the frequency uncertainties $\left(\sigma_{\mathrm{r}, \text { freq }}\right)$; as expected from Figure 1, the uncertainty ratios cluster around a value of 1. Bottom right: the distribution for the ratio between the corresponding ratio values. 

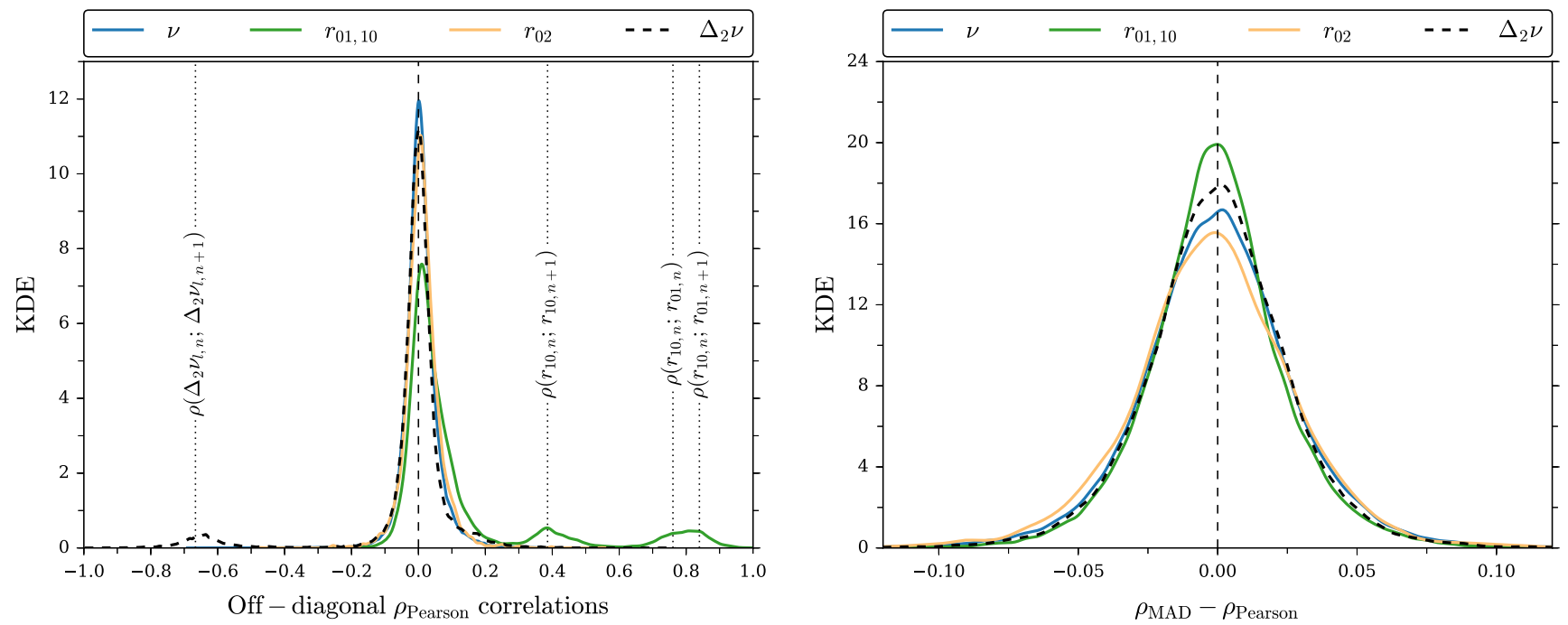

Figure 4. Left: the kernel density estimates of the off-diagonal elements of the correlation matrices for mode frequencies and frequency difference ratios (see the legend). A few of the expected correlations for $r_{01,10}$ ratios (dotted lines) and $\Delta_{2} \nu$ values are indicated. Right: a comparison between correlations calculated using the robust statistics method described in the original paper $\left(\rho_{\mathrm{MAD}}\right)$ and those estimated using the Pearson product-moment method $\left(\rho_{\text {Pearson }}\right)$.

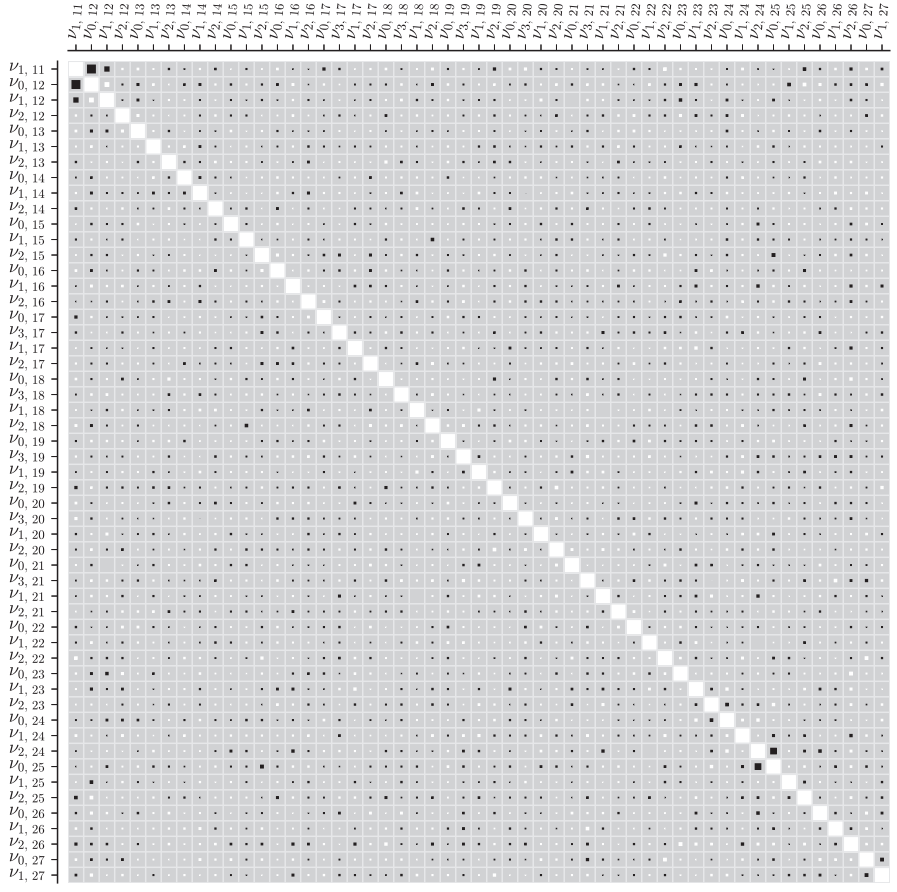

Frequencies in range $1334<\nu_{l, n}<2295(\mu \mathrm{Hz})$

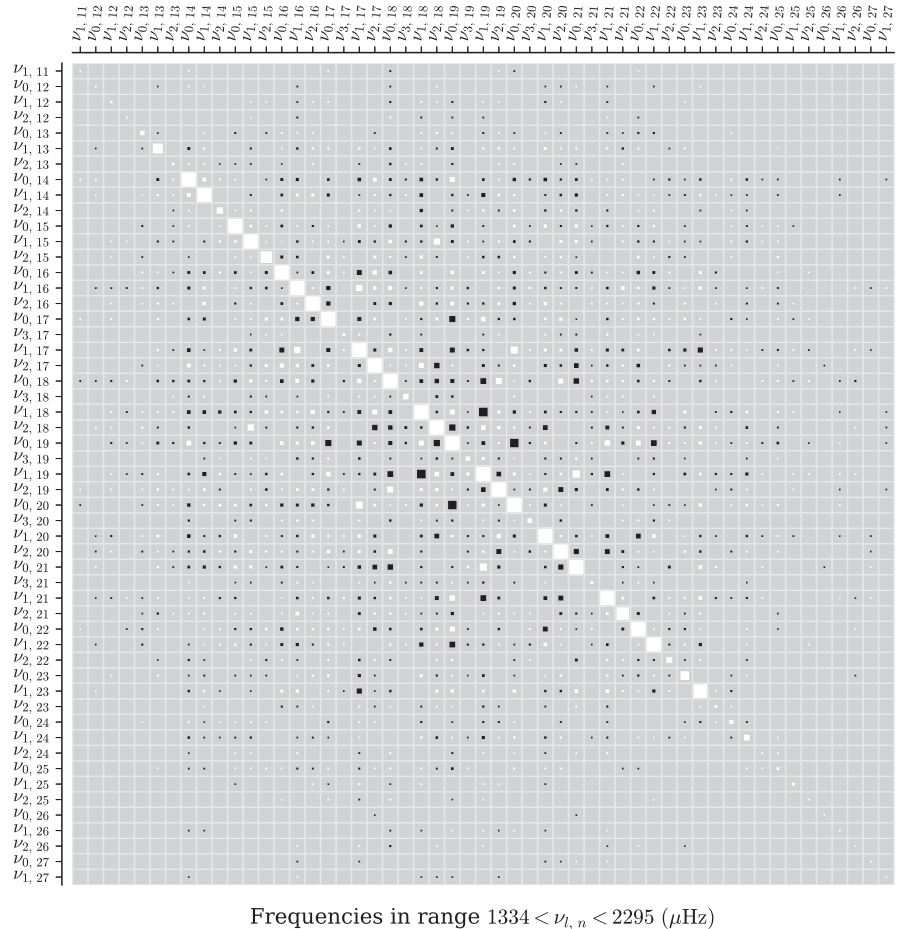

Frequencies in range $1334<\nu_{l, n}<2295(\mu \mathrm{Hz})$

Figure 5. Left: the correlation matrix for the mode frequencies of 16 Cyg A (KIC 12069424). The marker sizes indicate the absolute value of the correlation (between -1 and 1 ), and the color indicates the sign, white (black) representing a positive (negative) value. Right: the corresponding inverse covariance matrix, including only values with $\left|C_{i, j}^{-1}\right|>1$ and with values above $\left|C_{i, j}^{-1}\right|=100$ truncated to this value.

Corrected ratios. Concerning the updated $r_{01,10,02}$ values that followed the publication of the original paper, it has been suggested that the uncertainties on these were overestimated compared to expectations from the uncertainties on the individual mode frequencies (among others by Roxburgh 2017). We have identified the reason for this overestimation as a missing trimming in our post-processing of the MCMC chains, which is otherwise adopted for the parameters optimized in the peak-bagging (e.g., the individual mode frequencies) and in deriving $\Delta_{2} \nu$ values. The trimming concerns the removal of chains that for reasons unknown remain stationary during the entire MCMC run. By stationary, we mean that they did not move at all during the run (i.e., with an acceptance fraction of zero). Given that the initialization of the chains is done by sampling from the priors, the samples from these stationary chains simply represent a sparsely sampled version of the prior. Combined with the generous priors adopted for the individual frequencies - $\mathrm{a} 14 \mu \mathrm{Hz}$ wide top-hat prior regardless of the mode signal-to-noise ratio - these stationary chains have the 
Table 1

Extracted Mode Parameters and Quality Control for KIC 6225718 (Saxo2)

\begin{tabular}{|c|c|c|c|c|c|}
\hline$n$ & $l$ & $\begin{array}{l}\text { Frequency } \\
(\mu \mathrm{Hz})\end{array}$ & $\begin{array}{l}\text { Amplitude } \\
\text { (ppm) }\end{array}$ & $\begin{array}{l}\text { Line width } \\
\quad(\mu \mathrm{Hz})\end{array}$ & $\ln K$ \\
\hline 11 & 1 & $1351.14654_{-0.69757}^{+0.58628}$ & & & 2.2 \\
\hline 12 & 0 & $1407.22525_{-1.17619}^{+0.9539}$ & $0.78512_{-0.13082}^{+0.08601}$ & $2.50177_{-1.34340}^{+2.31218}$ & $>6$ \\
\hline 12 & 1 & $1454.25081_{-0.70081}^{+0.53443}$ & & & $>6$ \\
\hline 13 & 0 & $1510.10497_{-0.47674}^{+0.7020}$ & $0.98745_{-0.14508}^{+0.1646}$ & $2.50352_{-0.95426}^{+2.6690}$ & $>6$ \\
\hline 13 & 1 & $1558.44725_{-0.42460}^{+0.5435}$ & & & $>6$ \\
\hline 13 & 2 & $1605.68477_{-0.80743}^{+0.74438}$ & & & 1.45 \\
\hline 14 & 0 & $1615.12079_{-0.29009}^{+0.24304}$ & $1.16481_{-0.07484}^{+0.0712}$ & $2.60127_{-0.50727}^{+0.67106}$ & $>6$ \\
\hline 14 & 1 & $1664.08928_{-0.22534}^{+0.20516}$ & & & $>6$ \\
\hline 14 & 2 & $1711.39720_{-0.59557}^{+0.5037}$ & & & 3.14 \\
\hline 15 & 0 & $1720.35038_{-0.17192}^{+0.17913}$ & $1.46219_{-0.07172}^{+0.0687}$ & $2.30075_{-0.37050}^{+0.3793}$ & $>6$ \\
\hline 15 & 1 & $1769.65111_{-0.14426}^{+0.1452}$ & & & $>6$ \\
\hline 15 & 2 & $1816.18558_{-0.35636}^{+0.3438}$ & & & $>6$ \\
\hline 16 & 0 & $1825.41345_{-0.13041}^{+0.12475}$ & $1.89103_{-0.06605}^{+0.05765}$ & $2.19171_{-0.22374}^{+0.33798}$ & $>6$ \\
\hline 16 & 1 & $1873.87585_{-0.14081}^{+0.1348}$ & & & $>6$ \\
\hline 16 & 2 & $1919.96748_{-0.2588}^{+0.26436}$ & & & $>6$ \\
\hline 17 & 0 & $1929.04914_{-0.13812}^{+0.01225}$ & $2.28689_{-0.06048}^{+0.0564}$ & $2.84107_{-0.19777}^{+0.2986}$ & $>6$ \\
\hline 17 & 1 & $1977.34771_{-0.11998}^{+0.11272}$ & & & $>6$ \\
\hline 17 & 2 & $2023.79957_{-0.20783}^{+0.2128}$ & & & $>6$ \\
\hline 18 & 0 & $2032.67808_{-0.10642}^{+0.11221}$ & $2.76697_{-0.05294}^{+0.06538}$ & $2.67104_{-0.18240}^{+0.22980}$ & $>6$ \\
\hline 18 & 1 & $2081.57391_{-0.09209}^{+0.08960}$ & & & $>6$ \\
\hline 18 & 2 & $2128.61654_{-0.15773}^{+0.15407}$ & & & $>6$ \\
\hline 19 & 0 & $2137.58804_{-0.08658}^{+0.090724}$ & $3.14287_{-0.06114}^{+0.06464}$ & $2.49656_{-0.15248}^{+0.21005}$ & $>6$ \\
\hline 19 & 1 & $2186.89206_{-0.08988}^{+0.080099}$ & & & $>6$ \\
\hline 19 & 2 & $2234.70147_{-0.15890}^{+0.16414}$ & & & $>6$ \\
\hline 20 & 0 & $2243.41560_{-0.08141}^{+0.0845}$ & $3.47475_{-0.05883}^{+0.06700}$ & $2.22212_{-0.11738}^{+0.1581}$ & $>6$ \\
\hline 19 & 3 & $2281.61498_{-3.36130}^{+1.9694}$ & & & 3.01 \\
\hline 20 & 1 & $2293.05246_{-0.08844}^{+0.0895}$ & & & $>6$ \\
\hline 20 & 2 & $2340.63219_{-0.15687}^{+0.16692}$ & & & $>6$ \\
\hline 21 & 0 & $2349.63870_{-0.09205}^{+0.08380}$ & $3.46120_{-0.06114}^{+0.07072}$ & $2.61076_{-0.17082}^{+0.16363}$ & $>6$ \\
\hline 20 & 3 & $2385.56616_{-1.15569}^{+0.92700}$ & & & 3.94 \\
\hline 21 & 1 & $2399.38901_{-0.09518}^{+0.08395}$ & & & $>6$ \\
\hline 21 & 2 & $2446.70610_{-0.15376}^{+0.1557}$ & & & $>6$ \\
\hline 22 & 0 & $2455.69156_{-0.10461}^{+0.1054}$ & $3.46269_{-0.06289}^{+0.07197}$ & $3.03284_{-0.22768}^{+0.1810}$ & $>6$ \\
\hline 21 & 3 & $2493.07764_{-1.64119}^{+1.24010}$ & & & 3.66 \\
\hline 22 & 1 & $2505.34180_{-0.11043}^{+0.1044}$ & & & $>6$ \\
\hline 22 & 2 & $2552.85151_{-0.23715}^{+0.2216}$ & & & $>6$ \\
\hline 23 & 0 & $2561.29123_{-0.14098}^{+0.14766}$ & $3.17720_{-0.05647}^{+0.0583}$ & $4.00522_{-0.18167}^{+0.2533}$ & $>6$ \\
\hline 22 & 3 & $2598.55372_{-1.65837}^{+1.5480}$ & & & 3.12 \\
\hline 23 & 1 & $2611.20028_{-0.13537}^{+0.013497}$ & & & $>6$ \\
\hline 23 & 2 & $2658.62676_{-0.31997}^{+0.3268}$ & & & $>6$ \\
\hline 24 & 0 & $2666.48683_{-0.21998}^{+0.24134}$ & $2.72433_{-0.04411}^{+0.0567}$ & $5.23120_{-0.33358}^{+0.2623}$ & $>6$ \\
\hline 24 & 1 & $2717.47119_{-0.17163}^{+0.018160}$ & & & $>6$ \\
\hline 24 & 2 & $2765.04881_{-0.39587}^{+0.3953}$ & & & $>6$ \\
\hline 25 & 0 & $2773.05505_{-0.31397}^{+0.2957}$ & $2.40972_{-0.05643}^{+0.05210}$ & $6.75207_{-0.51121}^{+0.3896}$ & $>6$ \\
\hline 25 & 1 & $2824.15430_{-0.26309}^{+0.2650}$ & & & $>6$ \\
\hline 25 & 2 & $2872.28010_{-0.54465}^{+0.04493}$ & & & $>6$ \\
\hline 26 & 0 & $2879.34263_{-0.50316}^{+0.5586}$ & $1.95701_{-0.05736}^{+0.055}$ & $7.60008_{-0.81438}^{+0.6281}$ & $>6$ \\
\hline 26 & 1 & $2931.24401_{-0.34015}^{+0.3515}$ & & & $>6$ \\
\hline 26 & 2 & $2978.49401_{-0.84289}^{+0.8009}$ & & & 3.78 \\
\hline 27 & 0 & $2987.14941_{-0.48721}^{+0.48707}$ & $1.69613_{-0.06104}^{+0.04189}$ & $7.53133_{-0.74384}^{+0.8749}$ & $>6$ \\
\hline 27 & 1 & $3038.66527_{-0.52674}^{+0.51048}$ & & & $>6$ \\
\hline 27 & 2 & $3084.55010_{-1.58643}^{+1.37108}$ & & & 1.45 \\
\hline 28 & 0 & $3092.79984_{-0.92102}^{+0.88165}$ & $1.46461_{-0.06021}^{+0.06604}$ & $8.85413_{-1.10989}^{+1.43941}$ & $>6$ \\
\hline 28 & 1 & $3145.65332_{-0.62070}^{+0.6133}$ & & & 4.92 \\
\hline 28 & 2 & $3194.64154_{-1.26689}^{+1.68277}$ & & & 2.18 \\
\hline 29 & 0 & $3204.41215_{-0.92847}^{+0.88028}$ & $0.98137_{-0.10104}^{+0.07515}$ & $5.86594_{-1.40753}^{+2.75240}$ & 3.81 \\
\hline 29 & 1 & $3251.95767_{-1.47095}^{+1.1094}$ & & & 4.2 \\
\hline 29 & 2 & $3302.58742_{-2.57039}^{+2.1579}$ & & & 1.22 \\
\hline 30 & 0 & $3314.17150_{-2.02486}^{+2.06746}$ & $1.11864_{-0.07921}^{+0.0736}$ & $11.64170_{-1.65222}^{+1.5543}$ & 3.81 \\
\hline
\end{tabular}

Note. The complete table set (66 tables) is available in the online journal.

(This table is available in its entirety in machine-readable form.) 
Table 2

Example of Calculated Mode Frequency Difference Ratios $r_{01,10,02}(n)$ (Equation (3.16) in Lund et al. 2017) for KIC 6225718 (Saxo2)

\begin{tabular}{|c|c|c|}
\hline Ratio type & $n$ & Ratio \\
\hline$r_{02}$ & 14 & $0.08927_{-0.00778}^{+0.00748}$ \\
\hline$r_{02}$ & 15 & $0.08474_{-0.00484}^{+0.00599}$ \\
\hline$r_{02}$ & 16 & $0.08850_{-0.00332}^{+0.00354}$ \\
\hline$r_{02}$ & 17 & $0.08777_{-0.00270}^{+0.00276}$ \\
\hline$r_{02}$ & 18 & $0.08517_{-0.00217}^{+0.00230}$ \\
\hline$r_{02}$ & 19 & $0.08519_{-0.00165}^{+0.00169}$ \\
\hline$r_{02}$ & 20 & $0.08210_{-0.00160}^{+0.00163}$ \\
\hline$r_{02}$ & 21 & $0.08468_{-0.00175}^{+0.00159}$ \\
\hline$r_{02}$ & 22 & $0.08484_{-0.00171}^{+0.00173}$ \\
\hline$r_{02}$ & 23 & $0.07971_{-0.00241}^{+0.00229}$ \\
\hline$r_{02}$ & 24 & $0.07403_{-0.00365}^{+0.00368}$ \\
\hline$r_{02}$ & 25 & $0.07500_{-0.00415}^{+0.00447}$ \\
\hline$r_{02}$ & 26 & $0.06611_{-0.00667}^{+0.00658}$ \\
\hline$r_{02}$ & 27 & $0.08030_{-0.00840}^{+0.00803}$ \\
\hline$r_{02}$ & 28 & $0.07675_{-0.01179}^{+0.01314}$ \\
\hline$r_{02}$ & 29 & $0.09203_{-0.01412}^{+0.01445}$ \\
\hline$r_{10}$ & 12 & $0.04121_{-0.00939}^{+0.00961}$ \\
\hline$r_{01}$ & 13 & $0.03821_{-0.00545}^{+0.00794}$ \\
\hline$r_{10}$ & 13 & $0.03708_{-0.00425}^{+0.00658}$ \\
\hline$r_{01}$ & 14 & $0.03647_{-0.00313}^{+0.00314}$ \\
\hline$r_{10}$ & 14 & $0.03483_{-0.00217}^{+0.00225}$ \\
\hline$r_{01}$ & 15 & $0.03276_{-0.00181}^{+0.00168}$ \\
\hline$r_{10}$ & 15 & $0.03228_{-0.00150}^{+0.000150}$ \\
\hline$r_{01}$ & 16 & $0.03325_{-0.00138}^{+0.00137}$ \\
\hline$r_{10}$ & 16 & $0.03326_{-0.00138}^{+0.00134}$ \\
\hline$r_{01}$ & 17 & $0.03322_{-0.00128}^{+0.00129}$ \\
\hline$r_{10}$ & 17 & $0.03303_{-0.00120}^{+0.00125}$ \\
\hline$r_{01}$ & 18 & $0.03239_{-0.00113}^{+0.00104}$ \\
\hline$r_{10}$ & 18 & $0.03262_{-0.00094}^{+0.00101}$ \\
\hline$r_{01}$ & 19 & $0.03293_{-0.00094}^{+0.00088}$ \\
\hline$r_{10}$ & 19 & $0.03311_{-0.00090}^{+0.00082}$ \\
\hline$r_{01}$ & 20 & $0.03288_{-0.00079}^{+0.00085}$ \\
\hline$r_{10}$ & 20 & $0.03251_{-0.00085}^{+0.00087}$ \\
\hline$r_{01}$ & 21 & $0.03196_{-0.00086}^{+0.00091}$ \\
\hline$r_{10}$ & 21 & $0.03136_{-0.00087}^{+0.00092}$ \\
\hline$r_{01}$ & 22 & $0.03086_{-0.00097}^{+0.00104}$ \\
\hline$r_{10}$ & 22 & $0.02992_{-0.00119}^{+0.0011}$ \\
\hline$r_{01}$ & 23 & $0.02804_{-0.00150}^{+0.00134}$ \\
\hline$r_{10}$ & 23 & $0.02506_{-0.00158}^{+0.00157}$ \\
\hline$r_{01}$ & 24 & $0.02190_{-0.00202}^{+0.00183}$ \\
\hline$r_{10}$ & 24 & $0.02109_{-0.00225}^{+0.00220}$ \\
\hline$r_{01}$ & 25 & $0.02068_{-0.00274}^{+0.00288}$ \\
\hline$r_{10}$ & 25 & $0.01877_{-0.00343}^{+0.00338}$ \\
\hline$r_{01}$ & 26 & $0.01709_{-0.00416}^{+0.00402}$ \\
\hline$r_{10}$ & 26 & $0.01819_{-0.00391}^{+0.00419}$ \\
\hline$r_{01}$ & 27 & $0.01781_{-0.00486}^{+0.00461}$ \\
\hline$r_{10}$ & 27 & $0.01277_{-0.00653}^{+0.0069}$ \\
\hline$r_{01}$ & 28 & $0.01303_{-0.00697}^{+0.00718}$ \\
\hline$r_{10}$ & 28 & $0.02730_{-0.00657}^{+0.00658}$ \\
\hline$r_{01}$ & 29 & $0.05099_{-0.01214}^{+0.01314}$ \\
\hline
\end{tabular}

Note. The complete table set (66 tables) is available in the online journal.

(This table is available in its entirety in machine-readable form.)

effect of increasing the uncertainties on frequency difference ratios $r_{01,10,02}$. For the updated set of derived parameters, we thus still use the MCMC chains to form distributions for the derived parameters and estimate their value from the distribution median and uncertainties as the $68 \%$ highest probability density, as was done in Lund et al. (2017). 
Table 3

Example of Calculated Second Differences $\Delta_{2} \nu(n, l)$ (Equation (3.20) in Lund et al. 2017) for KIC 6225718 (Saxo2)

\begin{tabular}{|c|c|c|}
\hline$n$ & $l$ & $\begin{array}{c}\Delta_{2} \nu \\
(\mu \mathrm{Hz})\end{array}$ \\
\hline 12 & 1 & $1.49165_{-1.86861}^{+1.43042}$ \\
\hline 13 & 0 & $1.64320_{-1.65225}^{+2.97338}$ \\
\hline 13 & 1 & $1.19765_{-1.23010}^{+1.49066}$ \\
\hline 14 & 0 & $0.36802_{-1.08998}^{+0.82532}$ \\
\hline 14 & 1 & $0.13171_{-0.91027}^{+0.53955}$ \\
\hline 14 & 2 & $-1.08414_{-1.27950}^{+1.54658}$ \\
\hline 15 & 0 & $-0.22732_{-0.41376}^{+0.51838}$ \\
\hline 15 & 1 & $-1.27154_{-0.45265}^{+0.33595}$ \\
\hline 15 & 2 & $-0.96308_{-0.97206}^{+0.92238}$ \\
\hline 16 & 1 & $-0.76486_{-0.32231}^{+0.34620}$ \\
\hline 16 & 2 & $0.13943_{-0.78078}^{+0.60583}$ \\
\hline 16 & 0 & $-1.34692_{-0.40027}^{+0.25397}$ \\
\hline 17 & 0 & $0.01785_{-0.32003}^{+0.27051}$ \\
\hline 17 & 2 & $0.96664_{-0.50551}^{+0.56737}$ \\
\hline 17 & 1 & $0.69395_{-0.19823}^{+0.37672}$ \\
\hline 18 & 2 & $1.24972_{-0.40856}^{+0.39754}$ \\
\hline 18 & 1 & $1.13328_{-0.27680}^{+0.20918}$ \\
\hline 18 & 0 & $1.30380_{-0.28942}^{+0.25241}$ \\
\hline 19 & 2 & $-0.15373_{-0.42360}^{+0.39145}$ \\
\hline 19 & 1 & $0.82087_{-0.18954}^{+0.26296}$ \\
\hline 19 & 0 & $0.88559_{-0.20960}^{+0.24790}$ \\
\hline 20 & 2 & $0.13226_{-0.37283}^{+0.40859}$ \\
\hline 20 & 3 & $2.59020_{-2.76908}^{+4.75113}$ \\
\hline 20 & 1 & $0.14657_{-0.18242}^{+0.23765}$ \\
\hline 20 & 0 & $0.42375_{-0.22866}^{+0.19144}$ \\
\hline 21 & 3 & $-1.67592_{-3.51350}^{+3.17249}$ \\
\hline 21 & 1 & $-0.36648_{-0.24028}^{+0.20448}$ \\
\hline 21 & 2 & $0.15998_{-0.48495}^{+0.32524}$ \\
\hline 21 & 0 & $-0.15729_{-0.23049}^{+0.21404}$ \\
\hline 22 & 1 & $-0.09149_{-0.27147}^{+0.27138}$ \\
\hline 22 & 2 & $-0.42210_{-0.50012}^{+0.62788}$ \\
\hline 22 & 0 & $-0.44749_{-0.26079}^{+0.27197}$ \\
\hline 23 & 2 & $0.42624_{-0.53187}^{+1.05611}$ \\
\hline 23 & 0 & $-0.44262_{-0.34538}^{+0.42259}$ \\
\hline 23 & 1 & $0.38138_{-0.30050}^{+0.37274}$ \\
\hline 24 & 0 & $1.38224_{-0.61381}^{+0.53582}$ \\
\hline 24 & 2 & $0.71863_{-0.95902}^{+1.08429}$ \\
\hline 24 & 1 & $0.39741_{-0.40593}^{+0.46815}$ \\
\hline 25 & 0 & $-0.20075_{-0.96242}^{+0.72650}$ \\
\hline 25 & 2 & $-1.26870_{-1.16745}^{+1.55549}$ \\
\hline 25 & 1 & $0.46562_{-0.68934}^{+0.58511}$ \\
\hline 26 & 2 & $-0.14531_{-1.95515}^{+2.12575}$ \\
\hline 26 & 0 & $1.50744_{-1.26373}^{+1.17357}$ \\
\hline 26 & 1 & $0.35695_{-0.89877}^{+0.85148}$ \\
\hline 27 & 0 & $-1.98217_{-1.70044}^{+1.10727}$ \\
\hline 27 & 1 & $-0.34683_{-1.34396}^{+1.18152}$ \\
\hline 27 & 2 & $4.79796_{-4.22100}^{+2.77046}$ \\
\hline 28 & 2 & $-4.33698_{-3.40089}^{+6.79552}$ \\
\hline 28 & 1 & $-0.50516_{-2.17780}^{+1.67785}$ \\
\hline 28 & 0 & $6.76163_{-2.89273}^{+1.54528}$ \\
\hline 29 & 0 & $-1.46290_{-2.99269}^{+2.11596}$ \\
\hline
\end{tabular}

Note. The complete table set (66 tables) is available in the online journal.

(This table is available in its entirety in machine-readable form.) 
In Figure 1, we give an example of the influence of the stationary chains on a specific ratio distribution for the star KIC 9139151; as seen, the ratio distribution is the result of a wide background (the ratio prior), on top of which sits the main peak from nonstationary chains.

A comparison of the newly estimated $r_{01,10,02}$ values and associated uncertainties to those given in the original paper are given in Figure 2 for the star KIC 9139151. This particular star has a substantial change in ratio uncertainties (the middle panel), while the central parameter values are insignificantly changed (the right panel). As seen, the reduction in ratio uncertainty depends on the signal-to-noise ratio of the modes involved. Comparing the $r_{01,10,02}$ values from the original paper to those from the properly thinned chains for all targets, we find that these have not changed significantly (see Figure 3, top left). The bottom left panel of Figure 3 compares the $r_{01,10,02}$ uncertainties from the properly thinned chains with those obtained by sampling from the individual mode frequencies, assuming normal errors and no correlation between individual modes; the latter uncertainties thereby do not directly use the MCMC chains to calculate ratios and uncertainties, and would be the typical approach taken if only frequency values were given. From slight asymmetries that might occur in the mode frequency distributions the ratio values obtained directly from such central tendency frequency values will naturally differ to some extent from those obtained from the chains of the modes. Typically, the difference will be largest for the values that have the largest uncertainties. The bottom right panel of Figure 3 compares the $r_{01,10,02}$ values as estimated from the properly thinned chains with those obtained by sampling from the individual mode frequencies.

Updated $\Delta_{2} \nu_{l=3}$. In the original paper, the $\Delta_{2} \nu$ values for $l=3$ modes were incorrectly estimated; in total, the $\Delta_{2} \nu_{l=3}$ values of 12 stars are affected by this. For convenience, we provide this erratum with the full updated lists of $\Delta_{2} \nu$ values for all stars.

Additional. We note that the tick labels in Figure 6 of Lund et al. (2017) are given in the incorrect order; rather than giving all $r_{01}$ labels, followed by all $r_{10}$ labels, these should be mixed and given as a function of increasing central radial order.

We also note that the " $a$ " and " $b$ " column labels in Table 4 of Lund et al. (2017) should be swapped.

Correlations and covariances. We provide the covariance matrices for the individual mode frequencies $r_{01,10,02}$ and $\Delta_{2} \nu$ values of the sample. The original paper did not provide these, it only gave a visual representation of the $r_{01,10,02}$ covariance matrix for a specific star. Instead of calculating the covariance matrices using the robust statistics method described in the original paper ( $\left.\rho_{\mathrm{MAD}}\right)$, we have now opted for using the more standard Pearson product-moment correlation estimation. A reason for switching to the Pearson's method is that the robust statistics method was found to not always result in positive-definite covariance matrices.

In the left panel of Figure 4, we show the distributions for the off-diagonal elements of the correlation matrices, and also indicate a few of the expected correlations for the derived parameters. The right panel of Figure 4 shows the distribution for the difference in correlation value from the Pearson product-moment correlation estimation instead of the $\rho_{\mathrm{MAD}}$ measure. As seen, the method adopted for estimating the correlation has an influence at the level of $\rho= \pm 0.05$, hence correlation values with $|\rho|<0.05$ should be adopted cautiously. We note that the relatively large correlations between some neighboring elements of the correlation matrix, and the overall small uncertainties on the ratios, results in an inverse covariance matrix with very large values varying in sign between positive and negative-the absolute value of $\chi^{2}$ derived from comparison with another set of ratios should therefore be interpreted with care.

In Figure 5, we provide a representation of the frequency correlation matrix (the left panel) and the corresponding inverse covariance matrix $\left(C^{-1}\right.$, the right panel) for $16 \mathrm{Cyg}$ A (KIC 12069424). All points along the diagonals of the $C^{-1}$ matrices are positive, and all covariance matrices (and their inverse) are positive-definite in that all eigenvalues are positive. A further check of the latter was made by testing that a Cholesky decomposition could be performed, which requires the matrix to be positive-definite. We note that the correlation matrices displayed in Roxburgh (2017), which regrettably included the stationary chains from the MCMC analysis, were shared as part of a private communication and have never been used in an analysis or appeared in the public domain.

We are grateful to Ian Roxburgh for pointing out the larger than expected ratio uncertainties (Roxburgh 2017), which made us aware of the missing MCMC post-processing for the estimation of frequency difference ratios. We thank the referee for comments that helped improve the erratum.

\section{ORCID iDs}

Mikkel N. Lund (i) https://orcid.org/0000-0001-9214-5642

Víctor Silva Aguirre (iD https://orcid.org/0000-0002-6137-903X

Guy R. Davies (iD https://orcid.org/0000-0002-4290-7351

William J. Chaplin (i) https://orcid.org/0000-0002-5714-8618 Jørgen Christensen-Dalsgaard (ib https://orcid.org/0000-00015137-0966

Günter Houdek (iD https://orcid.org/0000-0003-1819-810X Timothy R. White (1) https://orcid.org/0000-0002-6980-3392 Timothy R. Bedding (i) https://orcid.org/0000-0001-5943-1460

Warrick H. Ball (1) https://orcid.org/0000-0002-4773-1017
Daniel Huber (iD https://orcid.org/0000-0001-8832-4488

H. M. Antia (1) https://orcid.org/0000-0001-7549-9684

David W. Latham (i) https://orcid.org/0000-0001-9911-7388

Kuldeep Verma (10) https://orcid.org/0000-0003-0970-6440

Sarbani Basu (1D https://orcid.org/0000-0002-6163-3472

Luca Casagrande (iD https://orcid.org/0000-0003-2688-7511

\section{References}

Lund, M. N., Silva Aguirre, V., Davies, G. R., et al. 2017, ApJ, 835, 172 Roxburgh, I. W. 2017, A\&A, 604, A42 